\title{
GMR
}

\section{Complete mitochondrial genome sequence and gene organization of Chinese indigenous chickens with phylogenetic considerations}

\author{
F.P. Zhao ${ }^{1,2 *}$, H.Y. Fan ${ }^{2,3 *}$, G.H. $\mathrm{Li}^{4}$ and B.K. Zhang ${ }^{1}$ \\ ${ }^{1}$ State Key Laboratory of Animal Nutrition, \\ College of Animal Science and Technology, China Agricultural University, \\ Key Laboratory of Feed Safety and Bioavailability, Ministry of Agriculture, \\ Beijing, China \\ ${ }^{2}$ Institute of Animal Sciences, Chinese Academy of Agricultural Sciences, \\ Beijing, China \\ ${ }^{3}$ College of Animal Science and Technology, Gansu Agricultural University, \\ Lanzhou, Gansu, China \\ ${ }^{4}$ Poultry Institute, Chinese Academy of Agricultural Sciences, Yangzhou, \\ Jiangsu, China
}

*These authors contributed equally to this study.

Corresponding author: B.K. Zhang

E-mail: bingkunzhang@126.com

Genet. Mol. Res. 15 (2): gmr. 15028200

Received August 8, 2015

Accepted November 26, 2015

Published July 15, 2016

DOI http://dx.doi.org/10.4238/gmr.15028200

ABSTRACT. In this study, we sequenced the complete mitochondrial DNA of Chinese indigenous Jinhu Black-bone and Rugao chickens. The two chicken mitochondrial genomes were deposited in GenBank under accession Nos. KP742951 and KR347464, respectively. The complete mitochondrial genomes of Jinhu Black-bone and Rugao chickens were sequenced and found to span 16,785 and 16,786 bp, respectively, and consisted of 22 tRNA genes, two rRNA genes (12S rRNA and 16S rRNA), 13 protein-coding genes, and one control region (D-loop). The majority of genes were positioned on the H-strand, 
and the ND6 and eight tRNA genes were found to be encoded on the L-strand. The mitogenomes showed a similar gene order to that of the published Gallus gallus genome, as neither included a control region. The overall base composition of the genome of the two chickens was $\mathrm{A}=30.22 / 30.28 \%, \mathrm{G}=13.57 / 13.49 \%, \mathrm{~T}=23.74 / 23.76 \%$, and $\mathrm{C}=$ $32.48 / 32.48 \%$. Nucleotide skewness of the coding strands of the two chicken genomes (AT-skew $=0.12, \mathrm{GC}$-skew $=-0.41)$ was biased towards T and G. Phylogenetic analysis revealed 29 subspecies, and the molecular genetic relationship among the 29 subspecies was identical to that of traditional taxonomy.

Key words: Chicken; Mitochondrial genome; Phylogenetic relationship

\section{INTRODUCTION}

Mitochondrial (mt) genomes are short in length, as they include short intergenic regions and lack introns. mtDNA has been used extensively as a genetic marker for phylogenetic analyses and is an ideal model of gene rearrangement and genome evolution. Complete mtDNA genomes have been reported in many studies on chicken, relying on the (partial) control region sequence (CR; displacement loop [D-loop]: nucleotide positions 1-1232; NC_007235). However, the relatively small size of the $\mathrm{CR}$ limits the resolution of mtDNA phylogeny. Because the mutation rate in this region is higher than in coding regions, high levels of recurrent mutations can blur the structure of the matrilineal genealogy. The D-loop is found in the main non-coding region of the mtDNA molecule in the CR or D-loop region (Pereira et al., 2004). mtDNA is a major control site for mtDNA expression and is important in maternal inheritance. This region varies in different populations and contains essential transcription and replication elements that can be used to investigate maternal inheritance and human evolution.

Jinhu Black-bone and Rugao chickens are indigenous Chinese breeds distributed in southeast China, and are bred for different purposes. The Jinhu Black-bone chicken is a dualpurpose chicken bred for ornamental value, whereas the Rugao Yellow chicken is bred for its meat and eggs. Therefore, it is important to obtain the complete mitogenome sequences to resolve their phylogenetic positions and interrelationships within Gallus. In the present study, we determined the complete mtDNA sequence of these two chicken breeds and described the organization of the genome, nucleotide composition, gene order, and codon use. Furthermore, we analyzed the molecular phylogenetic relationships between these and a further 27 chicken breeds. Our results will be useful for studies on population genetic structure, stock identification, evolution and phylogeny, and conservation genetics.

\section{MATERIAL AND METHODS}

\section{Ethics statement}

All animals used in the study were housed and handled following the guidelines for experimental animals established by the Council of China. Experimental procedures were approved and conducted in accordance with the guidelines of the China Agricultural University Animal Care and Use Committee. 


\section{Sample collection and DNA extraction}

Two Jinhu Black-bone chickens and two Rugao yellow chickens were obtained from local conservation farms in Jiangsu, China. All chickens were young, healthy females. Blood samples $(\sim 10 \mathrm{~mL})$ were drawn from the jugular vein by a licensed veterinarian and placed in K3 EDTA vacuum tubes. Total genomic DNA was extracted from all samples using the Qiagen DNeasy Blood \& Tissue Kit (Valencia, CA, USA) and stored at $-20^{\circ} \mathrm{C}$ until use.

\section{Polymerase chain reaction (PCR) amplification and sequencing}

The full mitogenomes of Rugao and Jinhu Black-bone chickens were amplified by PCR in 23 overlapping fragments, using previously published conserved primers (Bao et al., 2008). PCR amplifications were performed in a total volume of $25 \mu \mathrm{L}$ containing $12.5 \mu \mathrm{L}$ premixed enzyme, $2.5 \mu \mathrm{L}$ DNA template, $1.0 \mu \mathrm{L}$ of each primer $(10 \mathrm{ppm})$, and $8.0 \mu \mathrm{L}$ sterile deionized water. The PCR protocol was as follows: initial denaturation at $94^{\circ} \mathrm{C}$ for $3 \mathrm{~min}$, followed by 35 cycles in an ABI 9700 Cycler (Applied Biosystems, Foster City, CA, USA) at $94^{\circ} \mathrm{C}$ for $30 \mathrm{~s}$ (denaturation), $60^{\circ} \mathrm{C}$ for $30 \mathrm{~s} \mathrm{(annealing),} 72^{\circ} \mathrm{C}$ for $1 \mathrm{~min}$ (extension), and a final extension at $72^{\circ} \mathrm{C}$ for $10 \mathrm{~min}$. The PCR products $(5 \mu \mathrm{L})$ were detected on $1 \%$ agarose gel electrophoresis to confirm the length of the amplified fragment. Each amplicon was purified using the QIAquick PCR Purification Kit (Qiagen) and subjected to automated sequencing using an ABI 3730 sequencer (Applied Biosystems).

Another dataset used in this study was collected from the National Center for Biotechnology Information (NCBI) and included 19 local Chinese and 8 Indian chicken breeds.

\section{Sequence annotation and genomic analysis}

All sequences were inspected and assembled using DNASTAR and DNAMAN7.0. Target sequences were corrected by BLAST on the NCBI database. Protein-coding genes were determined by sequence comparisons using specialized BLAST at the NCBI database site. Next, these 13 genes were checked with the known complete mtDNA sequence of closely related species, including Daweishan Mini chickens (Yan et al., 2016), Huang Lang chickens (Yu et al., 2016), Taoyuan chickens (Liu et al., 2016b, and Xuefeng black-boned chickens (Liu, et al., 2016a). Twenty-one tRNA genes and their anticodons were annotated online using tRNAscan-SE Search Server v.1.21 (http://lowelab.ucsc.edu/tRNAscan-SE/) (Lowe and Eddy, 1997). Translation initiation and termination codons were identified by comparison with the mitochondrial genomes of known Gallus gallus codons. The complete mitochondrial genome was annotated with the Sequin program. Nucleotide composition was calculated for the entire genome using the EditSeq program included in the Lasergene software package, and the $\mathrm{C}, \mathrm{G}$, $\mathrm{A}$, and $\mathrm{T}$ frequencies of the four bases were computed using the following formulas: GC-skew $=(\mathrm{G}-\mathrm{C}) /(\mathrm{G}+\mathrm{C})$ and AT-skew $=(\mathrm{A}-\mathrm{T}) /(\mathrm{A}+\mathrm{T})$.

\section{Phylogenetic analysis}

A GenBank clustering analysis was performed on all chicken breeds (Table 1). These datasets were used to construct the phylogenetic tree for the Chinese and Indian 
chicken breeds. The complete mitochondrial genomes of 27 other chicken breeds were downloaded from the GenBank database in order to elucidate the phylogenetic position of the Chinese chickens. The phylogenetic tree was reconstructed using the unweighted pair group method with arithmetic means (UPGMA) in MEGA 6.0 with 1000 bootstrap replicates.

\section{RESULTS}

\section{Complete mitochondrial genome DNA sequences}

The complete mitochondrial genome sequences of Rugao and Jinhu Black-bone chickens were determined by PCR analysis and deposited in GenBank under accession Nos. KP742951 and KR347464. Twenty-three primer sets were used to determine the mtDNA sequences of Rugao and Jinhu Black-bone chickens, which were 16,786 and 16,785 bp, respectively. These lengths were similar to those reported for other chickens, including Gushi (16,785 bp), Xianju (16,784 bp), Xuefeng (16,785 bp), and Hengshan Yellow (16,785 bp) chickens (http://www.ncbi.nlm.nih.gov/).

\section{Genome organization and structure}

The overall nucleotide composition was 30.28 and $30.22 \% \mathrm{~A}, 23.76$ and $23.74 \% \mathrm{~T}$, 32.48 and $32.48 \% \mathrm{C}$, and 13.49 and $13.57 \% \mathrm{G}$ in the order $\mathrm{C}>\mathrm{A}>\mathrm{T}>\mathrm{G}$ for the Rugao and Jinhu Black-bone chickens, respectively. The $\mathrm{A}+\mathrm{T}$ percentages $(54.04$ and $53.96 \%)$ were greater than the $\mathrm{G}+\mathrm{C}$ percentages (45.97 and $46.05 \%$ ). The mitochondrial gene arrangements of Jinhu Black-bone and Rugao yellow chicken are shown in Figures 1 and 2, respectively. The gene arrangement and transcriptional direction were similar to those of typical G. gallus mitogenomes (Desjardins and Morais, 1990). As shown in Figures 1 and 2, neither mtDNA genome contained a $\mathrm{CR}$, but both contained a single-extra cytidine base in the ND3 gene. The total lengths of the 13 protein-coding genes in Jinhu Black-bone and Rugao yellow chickens were 11,438 and $11,437 \mathrm{bp}$, respectively, accounting for 68.14 and $68.14 \%$ of the entire mitogenome. All 13 genes of the two chickens were located on heavy chains, with the exception of ND6, which was found to be located on a light chain. Only the cytochrome c oxidase subunit I (COI) gene used GTG as a start codon, whereas the other 12 genes used ATG. The COX3 and ND4 genes were inferred to end with an incomplete stop codon (T--); the ND2 gene stopped with TAG; the COI gene terminated with AGG; and the other proteincoding genes all used a TAA termination codon. The mitochondrial genomes of the Rugao yellow and Jinhu Black-bone chickens contained 22 tRNA genes, which ranged from 66 to $76 \mathrm{bp}$ in length; 14 were on a heavy chain and 8 were on a light chain (Table 1). The CRs of the Rugao yellow and Jinhu Black-bone chicken mitochondrial genomes were 1232-1233-bp long. Thirteen gene overlaps were found, of which three were on the H-strand and 10 were on the L-strand. Five intergenic spacers were detected; one spacer was on the $\mathrm{H}$-strand, and four were on the L-strand. The overlaps and intergenic spacers were all 1-9 bp in size at each site (Table 1). The longest overlap ( $9 \mathrm{bp}$ ) was located between tRNA-Leu and ND1, and the largest intergenic spacer of 9 bp occurred between ATP8 and ATP6. The overlap and intergenic spacers of Rugao yellow and Jinhu Black-bone chickens were of different lengths than those of the other chicken breeds (Wang et al., 2016). 


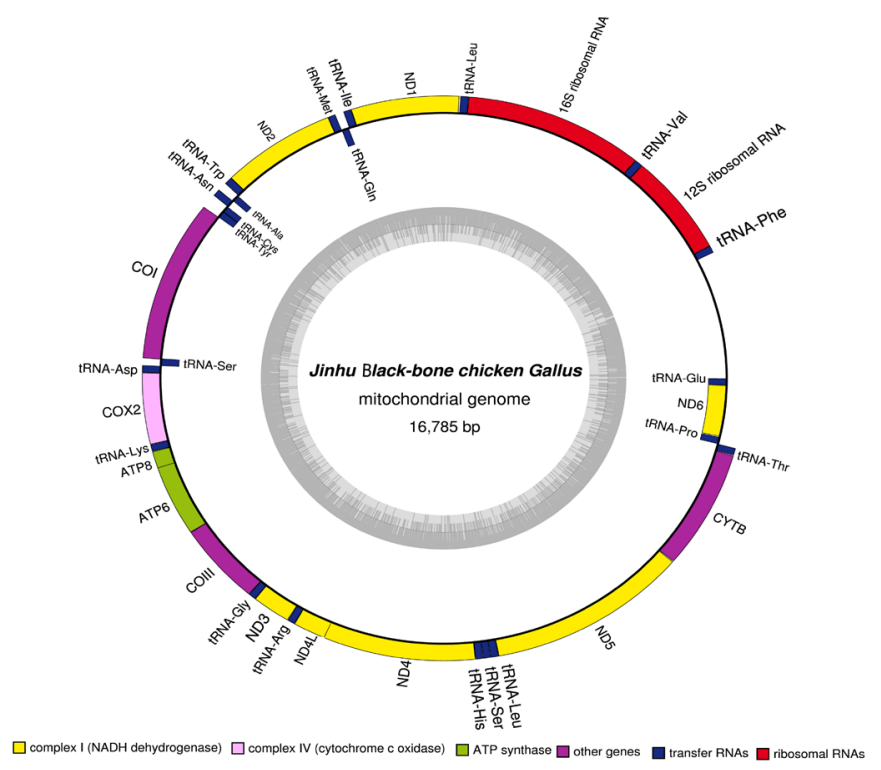

Figure 1. Mitochondrial gene arrangement in Jinhu Black-bone chickens.

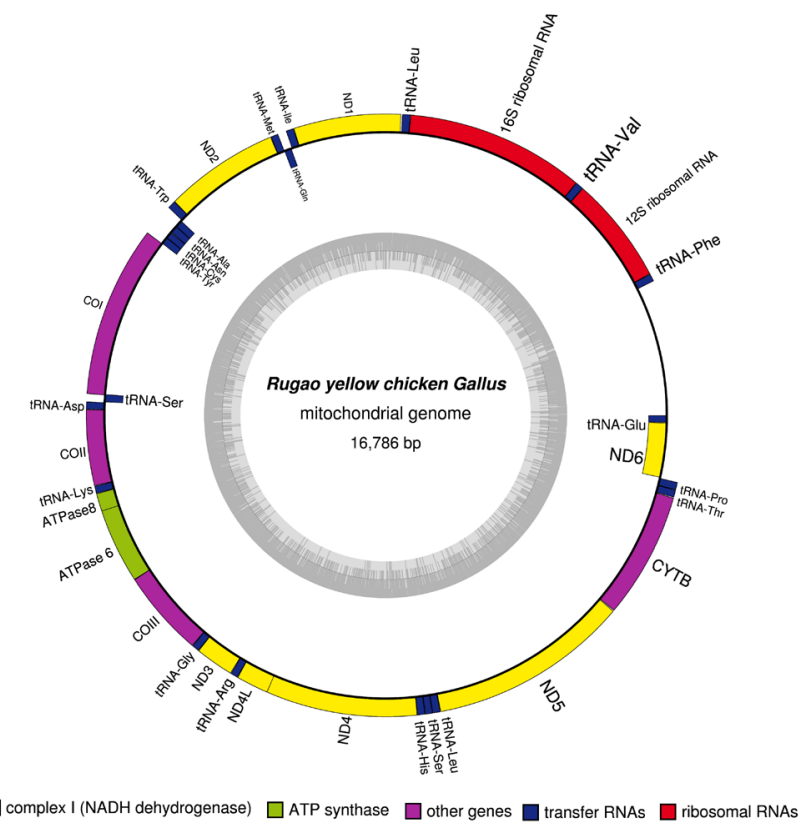

Figure 2. Mitochondrial gene arrangement in Rugao yellow chickens. 
Nucleotide skewness values for the G. gallus coding strands (AT-skew $=0.12$ and GC-skew $=-0.41)$ were biased towards A and C. Such an A-T rich pattern reflects a typical sequence feature of the vertebrate mitochondrial genome (Mayfield and McKenna, 1978; Hiendleder, et al., 1998). The nucleotide composition of the coding strand was biased towards $\mathrm{T}$ and $\mathrm{A}$ in $\mathrm{C}$, which is consistent with most G. gallus mitogenomes (Jiang et al., 2013).

Table 1. Complete chicken mitogenomes deposited in GenBank.

\begin{tabular}{l|l|c|c}
\hline No. & Species & GenBank accession No. & Length (bp) \\
\hline 1 & Gushi Chicken & GU261678.1 & 16,785 \\
\hline 2 & Chigulu Chicken & GU261719.1 & 16,785 \\
\hline 3 & Tengchongxue Chicken & GU261689 & 16,785 \\
\hline 4 & Nixi Chicken & KM81711.1 & 16,785 \\
\hline 5 & Dongan Black Chicken & KP681580 & 16,785 \\
\hline 6 & Guangxi partridge Chicken & GU261675.1 & 16,785 \\
\hline 7 & Xuefeng Chicken & NC_007235.1 & 16,785 \\
\hline 8 & Gallus gallus Spadiceus Chicken & GU261699 & 16,785 \\
\hline 9 & Wenshanshandi Chicken & KP681581 & 16,785 \\
\hline 10 & Guangxi Three-buff Chicken & GU261714 & 16,785 \\
\hline 11 & Jiangbian Chicken & GU261676 & 16,785 \\
\hline 12 & Wuding Chicken & DQ648776 & 16,788 \\
\hline 13 & Tibetan Chicken & KF981434.1 & 16,783 \\
\hline 14 & Taoyuan Chicken & GU261677 & 16,784 \\
\hline 15 & Xianju Chicken & GU261683 & 16,784 \\
\hline 16 & Tulufan Chicken & KF954727 & 16,784 \\
\hline 17 & Huanglang Chicken & KM433666.1 & 16,785 \\
\hline 18 & Cenxi Classical Three-buff Chicken & KM886937.1 & 16,786 \\
\hline 19 & Dongan Yellow Chicken & KP211422 & 16,785 \\
\hline 20 & Nicobari Brown Chicken & KP211424 & 16,775 \\
\hline 21 & Tellicherry Chicken & KP211418 & 16,775 \\
\hline 22 & Aseel Chicken & KP211419 & 16,775 \\
\hline 23 & Ghagus Chicken & KP211420 & 16,775 \\
\hline 24 & Haringhata Chicken & KP211425 & 16,775 \\
\hline 25 & Kadaknath Chicken & KP211421.1 & 16,775 \\
\hline 26 & Nicobari Black Chicken & KP211423.1 & 16,775 \\
\hline 27 & Red Jungle Fowl Chicken & & 16,775 \\
\hline & & & \\
\hline
\end{tabular}

\section{Phylogenetic analysis}

A phylogenetic tree, using the complete mtDNA and CRs of Rugao yellow and Jinhu Black-bone chickens, was constructed using the UPGMA method (Tamura and Nei, 1993) and included two datasets. The first dataset was provided from our sequences, and the second was downloaded from NCBI. A bootstrap consensus tree inferred from 1000 replicates was used to represent the taxonomy of the chickens (Felsenstein, 1985). Branches corresponding to partitions reproduced with $<50 \%$ bootstrap replicates collapsed. Evolutionary distances were computed using the maximum composite likelihood method (Tamura et al., 2007) and were in units of the number of base substitutions per site. The analysis involved 32 nucleotide sequences, and the first, second, third, and non-coding codon positions were included. All positions containing gaps and missing data were eliminated. Clustering analyses were conducted using MEGA6.0 (Tamura, et al., 2013).

The chicken matrilineal phylogeny was constructed using the complete mitochondrial genome (Figure 3) and CR (Figure 4). In addition to the two Chinese indigenous chicken breeds used in this study, we also downloaded the mitochondrial genome sequences of 27 other chicken breeds from NCBI, which are summarized in Table 2. The cluster tree results showed that the 29 chicken breeds could be divided into two groups using the complete 
chicken mitochondrial genomes. One group included the Chinese chicken breeds and the other group included the Indian chicken breeds. Additionally, the Chinese indigenous chickens were assigned into five groups based on the geographic region and skin color. The Wuding chicken was alone in a group, which may have occurred because local farmers do not buy chickens, instead selling chickens that they have bred over a long time.

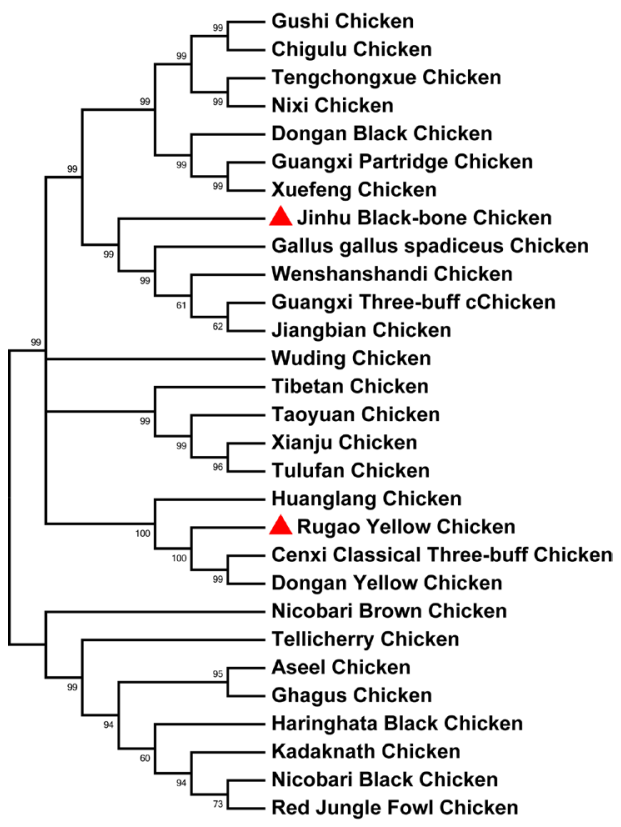

Figure 3. Evolutionary relationships between chicken breeds based on their complete mitochondrial genomes.

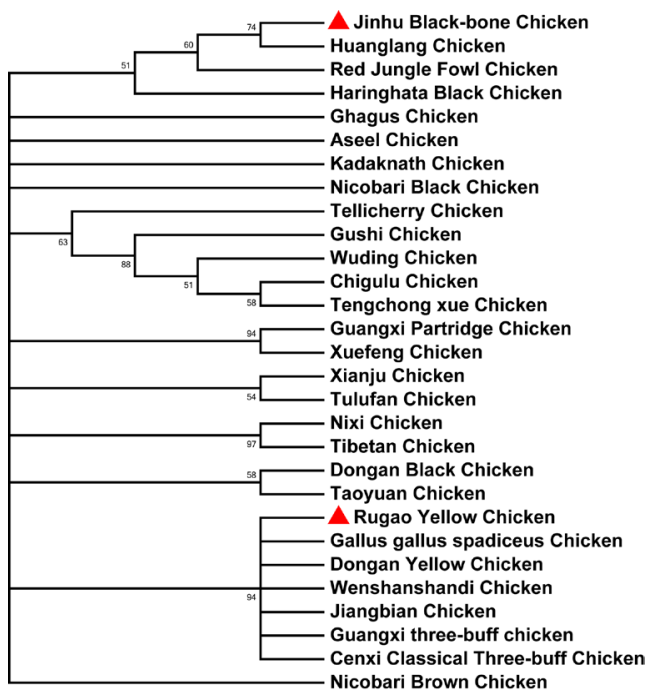

Figure 4. Evolutionary relationships between chicken breeds based on the mitogenome control region. 
Table 2. Characteristics of protein coding genes and structural RNAs in the mitochondrial genome of Rugao yellow and Jinhu Black-bone chickens.

\begin{tabular}{|c|c|c|c|c|c|c|c|c|}
\hline \multirow[t]{2}{*}{ Genes } & \multicolumn{2}{|c|}{ Position } & \multirow[t]{2}{*}{ Length (bp) } & \multicolumn{2}{|c|}{ Codons } & \multirow[t]{2}{*}{ Anti-codon } & \multirow{2}{*}{$\begin{array}{l}\text { Intergenic } \\
\text { nucleotide }\end{array}$} & \multirow[t]{2}{*}{ Strand } \\
\hline & Start & End & & Start & Stop & & & \\
\hline D-loop & $1 / 1$ & $1233 / 1232$ & 1233 & & & & & \\
\hline tRNA-Phe & $1234 / 1233$ & $1303 / 1302$ & $70 / 70$ & & & GAA & 0 & $\mathrm{H}$ \\
\hline rRNA & $1304 / 1302$ & $2278 / 2277$ & 975/976 & & & & 0 & $\mathrm{H}$ \\
\hline tRNA-Val & $2279 / 2278$ & $2351 / 2350$ & $73 / 73$ & & & TAC & 0 & $\mathrm{H}$ \\
\hline rRNA & $2352 / 2351$ & $3973 / 3972$ & $1622 / 1622$ & & & & 0 & $\mathrm{H}$ \\
\hline tRNA-Leu & $3974 / 3973$ & $4047 / 4046$ & $74 / 74$ & & & TAA & 9 & $\mathrm{H}$ \\
\hline ND1 & $4057 / 4056$ & $5031 / 5030$ & $975 / 975$ & ATG & TAA & & 0 & $\mathrm{H}$ \\
\hline tRNA-Ile & $5032 / 5031$ & $5103 / 5102$ & $72 / 72$ & & & GAT & 5 & $\mathrm{H}$ \\
\hline tRNA-Gln & $5109 / 5108$ & $5179 / 5178$ & $71 / 71$ & & & TTG & -1 & $\mathrm{~L}$ \\
\hline tRNA-Met & $5179 / 5178$ & $5247 / 5246$ & $69 / 69$ & & & CAT & 0 & $\mathrm{H}$ \\
\hline ND2 & $5248 / 5247$ & $6288 / 6287$ & $1041 / 1041$ & ATG & TAG & & 0 & $\mathrm{H}$ \\
\hline tRNA-Trp & $6287 / 6286$ & $6362 / 6361$ & $76 / 76$ & & & TCA & 0 & $\mathrm{H}$ \\
\hline tRNA-Ala & $6369 / 6368$ & $6441 / 6436$ & 69/69 & & & TGC & 0 & $\mathrm{~L}$ \\
\hline tRNA-Asn & $6441 / 6440$ & $6513 / 6512$ & $73 / 73$ & & & GTT & 1 & $\mathrm{~L}$ \\
\hline tRNA-Cys & $6515 / 6514$ & $6580 / 6579$ & $66 / 66$ & & & GCA & -1 & $\mathrm{~L}$ \\
\hline tRNA-Tyr & $6580 / 6579$ & $6650 / 6649$ & $71 / 71$ & & & GTA & 1 & $\mathrm{~L}$ \\
\hline $\mathrm{COI}^{*}$ & $6652 / 6651$ & $8202 / 8201$ & $1551 / 1551$ & GTG & AGG & & -7 & $\mathrm{H}$ \\
\hline tRNA-Ser & $8194 / 8193$ & $8268 / 8267$ & $75 / 75$ & & & TGA & 2 & $\mathrm{~L}$ \\
\hline tRNA-Asp & $8271 / 8270$ & $8339 / 8338$ & $69 / 69$ & & & GTC & 1 & $\mathrm{H}$ \\
\hline COX2 & $8341 / 8340$ & $9024 / 9023$ & 684/684 & ATG & TAA & & 1 & $\mathrm{H}$ \\
\hline tRNA-Lys & $9026 / 9025$ & $9093 / 9092$ & $68 / 68$ & & & TTT & 1 & $\mathrm{H}$ \\
\hline ATP8 & $9095 / 9094$ & $9259 / 9258$ & 209/209 & ATG & TAA & & -9 & $\mathrm{H}$ \\
\hline ATP6 & $9250 / 9249$ & $9933 / 99332$ & $684 / 684$ & ATG & TAA & & 0 & $\mathrm{H}$ \\
\hline $\mathrm{COX} 3$ & $9932 / 9932$ & $10716 / 10715$ & $785 / 784$ & ATG & T-- & & 0 & $\mathrm{H}$ \\
\hline tRNA-Gly & $10717 / 10716$ & $10785 / 10784$ & $69 / 69$ & & & TCC & 0 & $\mathrm{H}$ \\
\hline ND3 & $10786 / 10785$ & $11137 / 11136$ & $352 / 352$ & ATG & TAA & & 1 & $\mathrm{H}$ \\
\hline tRNA-Arg & $11139 / 11138$ & $11206 / / 11205$ & $68 / 68$ & & & TCG & 0 & $\mathrm{H}$ \\
\hline ND4L & $11207 / 11206$ & $11503 / 11502$ & $297 / 297$ & ATG & TAA & & -5 & $\mathrm{H}$ \\
\hline ND4 & $11497 / 11496$ & $12874 / 12873$ & $1378 / 1378$ & ATG & T-- & & 0 & $\mathrm{H}$ \\
\hline tRNA-His & $12875 / 12874$ & $12943 / 12492$ & $69 / 69$ & & & GTG & 0 & $\mathrm{H}$ \\
\hline tRNA-Ser & $12944 / 12944$ & $13010 / 13008$ & $67 / 65$ & & & GCT & 0 & $\mathrm{H}$ \\
\hline tRNA-Leu & $13011 / 13010$ & $13081 / 13080$ & $71 / 71$ & & & ATG & 0 & $\mathrm{H}$ \\
\hline ND5 & $13082 / 13081$ & $14899 / 14898$ & $1818 / 1817$ & ATG & TAA & & 4 & $\mathrm{H}$ \\
\hline Cyt b & $14904 / 14903$ & $16046 / 16045$ & $1142 / 1142$ & ATG & TAA & & 4 & $\mathrm{H}$ \\
\hline
\end{tabular}

\section{DISCUSSION}

The mtDNA sequences of the Rugao yellow and Jinhu Black-bone chickens were determined independently for each individual animal. The mtDNA sequences derived from the two local Chinese chickens matched and were registered in the NCBI database. The complete mtDNA sequences of the two chickens were 16,785 and 16,786 bp in size. Both sequences contained 13 protein-coding genes, 22 tRNA genes, two rRNA genes, and one CR. The genome organization and structure were the same as those of the three other chicken breeds (Gushi chicken, Xuefeng chicken, and Hengshan Yellow chicken). The sequence lengths differed slightly, mainly due to changes in the CR. The overall A $+\mathrm{T}$ contents of the Rugao yellow and Jinhu Black-bone chicken mtDNA genomes were 54.04 and $53.96 \%$, which were similar to those of other chicken breeds, such as Daweishan Mini chickens (Yan et al., 2016), Gallus domesticus Brisson (Wang et al., 2016), and Huanglang chickens (Yu et al., 2016). The Rugao yellow and Jinhu Black-bone chickens possessed 13 gene overlaps and five intergenic spacers, which differs from other chicken breeds (Liu et al., 2016a,b).

Organization of the CR, 13 protein-coding genes, 22 tRNA genes, and two rRNA genes of the Rugao yellow and Jinhu Black-bone chickens was identical to the reported organization in other chickens. The protein-coding genes started with ATG or GTG. The 13 protein-coding genes possessed a variety of termination codons, including TAA, TAG, 
AGG, and T--. The incomplete termination codon $(\mathrm{T})$ indicates that the TAA transcription process was terminated after the PolyA tail was affixed (Clayton, 1991). This stop codon is reported commonly in many G. gallus breeds. In this study, we describe the organization and structure of the mitochondrial genome of two local Chinese chicken breeds and determined the molecular evolutionary relationships between these Chinese indigenous chickens and Indian chicken breeds. Neighbor-joining, maximum-likelihood, and UPGMA trees constructed using the maximum composite likelihood method showed the same results. Therefore, in this report, only the UPGMA tree is presented.

In conclusion, the complete mitochondrial genome DNA sequences of Jinhu Blackbone and Rugao yellow chickens were determined to be 16,785 and 16,786 bp in length. Our results will be of value for studies on population genetic structure, stock identification, evolution and phylogeny, and conservation genetics of G. gallus and related chicken breeds.

\section{Conflicts of interest}

The authors declare no conflict of interest.

\section{ACKNOWLEDGMENTS}

Research supported by the Beijing Higher Education Young Elite Teacher Project and the State Key Laboratory of Animal Nutrition, Key Laboratory of Feed Safety and Bioavailability, Ministry of Agriculture (\#2004DA125184F1301).

\section{REFERENCES}

Bao H, Zhao C, Li J and Wu C (2008). Sequencing and alignment of mitochondrial genomes of Tibetan chicken and two lowland chicken breeds. Sci. China C Life Sci. 51: 47-51. http://dx.doi.org/10.1007/s11427-008-0005-0

Clayton DA (1991). Replication and transcription of vertebrate mitochondrial DNA. Annu. Rev. Cell Biol. 7: 453-478. http://dx.doi.org/10.1146/annurev.cb.07.110191.002321

Desjardins P and Morais R (1990). Sequence and gene organization of the chicken mitochondrial genome. A novel gene order in higher vertebrates. J. Mol. Biol. 212: 599-634. http://dx.doi.org/10.1016/0022-2836(90)90225-B

Felsenstein J (1985). Confidence limits on phylogenies: an approach using the bootstrap. Evolution 39: 783-791. http:// dx.doi.org/10.2307/2408678

Hiendleder S, Lewalski H, Wassmuth R and Janke A (1998). The complete mitochondrial DNA sequence of the domestic sheep (Ovis aries) and comparison with the other major ovine haplotype. J. Mol. Evol. 47: 441-448. http://dx.doi. org/10.1007/PL00006401

Jiang L, Wang G, Tan S, Gong S, et al. (2013). The complete mitochondrial genome sequence analysis of Tibetan argali (Ovis ammon hodgsoni): implications of Tibetan argali and Gansu argali as the same subspecies. Gene 521: 24-31. http://dx.doi.org/10.1016/j.gene.2013.03.049

Liu LL, Xie HB, Yang YS, Yu QF, et al. (2016a). The complete mitochondrial genome of the Xuefeng black-boned chicken. Mitochondrial DNA 27: 30-31. http://dx.doi.org/10.3109/19401736.2013.869679

Liu LL, Xie HB, Yu QF, He SP, et al. (2016b). Determination and analysis of the complete mitochondrial genome sequence of Taoyuan chicken. Mitochondrial DNA 27: 371-372. http://dx.doi.org/10.3109/19401736.2014.895991

Lowe TM and Eddy SR (1997). tRNAscan-SE: a program for improved detection of transfer RNA genes in genomic sequence. Nucleic Acids Res. 25: 955-964. http://dx.doi.org/10.1093/nar/25.5.0955

Mayfield JE and McKenna JF (1978). A-T rich sequences in vertebrate DNA. A possible explanation of q-banding in metaphase chromosomes. Chromosoma 67: 157-163. http://dx.doi.org/10.1007/BF00293173

Pereira L, Van Asch B and Amorim A (2004). Standardisation of nomenclature for dog mtDNA D-loop: a prerequisite for launching a Canis familiaris database. Forensic Sci. Int. 141:99-108. http://dx.doi.org/10.1016/j.forsciint.2003.12.014

Tamura K and Nei M (1993). Estimation of the number of nucleotide substitutions in the control region of mitochondrial 
DNA in humans and chimpanzees. Mol. Biol. Evol. 10: 512-526.

Tamura K, Dudley J, Nei M and Kumar S (2007). MEGA4: molecular evolutionary genetics analysis (MEGA) software version 4.0. Mol. Biol. Evol. 24: 1596-1599. http://dx.doi.org/10.1093/molbev/msm092

Tamura K, Stecher G, Peterson D, Filipski A, et al. (2013). MEGA6: molecular evolutionary genetics analysis version 6.0. Mol. Biol. Evol. 30: 2725-2729. http://dx.doi.org/10.1093/molbev/mst197

Wang S, Wang B, Wang F and Wu Z (2016). Complete mitochondrial genome of Gallus domesticus (Galliformes: Phasianidae). Mitochondrial DNA A DNA MappSeq Anal 27: 978-979. http://dx.doi.org/10.3109/19401736.2014. $\underline{926514}$

Yan ML, Ding SP, Ye SH, Wang CG, et al. (2016). The complete mitochondrial genome sequence of the Daweishan Mini chicken. Mitochondrial DNA 27: 138-139. http://dx.doi.org/10.3109/19401736.2013.878913

Yu QF, Liu LL, Fu CX, He SP, et al. (2016). The complete mitochondrial genome of the Huang Lang chicken. Mitochondrial DNA 27: 216-217. http://dx.doi.org/10.3109/19401736.2014.880895 